\title{
Development of an Application to Support WEB Navigation
}

\author{
Oscar Sanjuán Martínez, Vanessa Cejudo Mejías, Javier Parra, Andrés Castillo, \\ and Luis Joyanes Aguilar \\ Universidad Pontificia de Salamanca in Madrid \\ Paseo Juan XXIII, $n^{\circ}$ 3. Madrid. Spain \\ sanjuan@acm.org, vcejudo@upsaguate.org, \\ ljoyanes@upsaguate.org
}

\begin{abstract}
The Internet has evolved too rapidly, which has left us with no option but to leave many problems unsolved. In many occasions, this is due to the fact that this 'invention' was devised with a different purpose from what we understand as the Internet nowadays.
\end{abstract}

The Web aims to be a universally helpful utility.

Our Proposal consists of the development of a tool which makes the Web something more accessible to everybody, and it is aimed at solving language, understanding, and displaying problems, by building a new navigation interface based on icons.

\section{Background}

We are now witnessing the evolution of this universal instrument, which changes depending on users' needs. According to Ben Schnneiderman's words, the Internet is necessary as a means of achieving equality of opportunities. The information found on the Web is of growing general interest. Nowadays, there are many initiatives, which demand that both the contents and the design of Web pages from public entities are easily accessible [REVE02]. In order to permit equality of opportunities, Clement, A. and Shade, L.R. have pointed out seven major aspects: Transport Services, Devices, Software Tools, Contents Service, Server Access Forecast, Social Service, and Government.

Our tool stands out in the field of the software tools that aim the access to the Net to be improved.

\section{What Is SAN?}

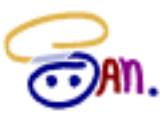

SAN - Sistema de Ayuda a la Navegación (Navigation Support System )

San is a system that adds a new navigation interface to any Web page; an interface based on the use of icons which aims to: 
- Facilitate access to the page contents by adding an icon bar that appears at the top of the page, in which, through the use of various icons, major topics are shown, along with the page resources.

- Filter information. Thanks to this navigation bar, we can view, in a quicker and more effective way, the contents and resources we need.

- Sort the information. The navigation bar will display the most important contents in the form of icons/ sounds, by means of a hierarchy of topics sorted in order of relevance.

- Soften language barriers. Icons are easily understood in any language, since they represent concepts, not words; thus, SAN could help to remove some of the linguistic obstacles that users encounter when navigating the Internet.

\section{How Does SAN Work?}

SAN interface is implemented separately from the page, and it does not modify it. SAN is able to dynamically generate the navigation bar and to incorporate it to the display of the Web. The SAN interface is made up of icons that mainly represent two things:

- Contents: That is, the most relevant concepts that the page refers to.

- Resources: Some specific elements that the page has, such as photos, files to be downloaded, and so on.

The navigation bar acts as a shortcut bar to the information that can be found on the page. When clicking one of the icons, the user can access either to that part of the Web where the concept is shown or to the resource in question.

\section{SAN's Advantages}

- The icons allow us to quickly perform Content based Navigation using the most universal language of all: images.

- SAN provides a faster, more intuitive navigation, since it synthesizes the contents of Web pages through the use of conceptual images. This allows the user to get an idea of the most relevant concepts of the page, with just a quick glance.

- It promotes the use of a universal language of icons, as well as its possible use as a standard.

- It allows for a faster navigational access, thanks to the discrimination of contents, leaving only those that are considered to be more relevant for the user.

- It organizes and structures the information of the Web site.

- It improves the usability of Websites. 


\section{Architecture and Operation}

\subsection{Architecture}

The first version of SAN has a repository with all the icons, concepts, rules and relationships needed to infer the concepts from each page. The present technology used to develop SAN has been ASP pages, with COM components that embed the system's behavior and intelligence. SAN downloads the desired page and analyses it in order to locate the concepts that appear on the page. Such concepts are sorted in order of relevance and appearance on the page; this prioritized list will be considered as a basis for the generation of the appropriate icon bar.

\subsection{Operation}

After login in the system, a Web page informs the user about the virtues of SAN and will also allow to type the URL of the page the user wants to visit.

Once this operation has been completed, SAN takes over and generates the corresponding bar, as we will see in the example that follows.

When the user clicks one of the icons of the intelligent bar, SAN generates a link to the area related to the concept pointed out by the icon. The significant text will be highlighted in order to catch the user's attention.

If a link is chosen, the user will be transmitted to the corresponding URL and SAN will rebuild the icon bar, in order to adapt it to the contents of the new page.

\section{Current and Future Work}

It is our contention to make of SAN a pioneering tool for the humanization of the Internet, making it more accessible for everyone, specially for those who can obtain more benefit from it: the disabled, the children, or people who just want to have a different kind of approach to the Net.

We are nowadays working on a new version of SAN, a totally upgraded one, which aims at solving some of the problems encountered, and one that takes a completely different approach to the development of the system, but maintaining its original objectives.

Currently, we are working on an off-line version of SAN, that would work like a spider and that would generate a conceptual database of pages, which will allow SAN not only to analyze the superficial information of the Web page, but also to perform more in-depth searches.

Our more immediate goals are:

- Migrating to the new application model.

- Increasing the update capability of the database.

- Taking a close look at possible learning mechanisms.

- Carrying out some tests in order to evaluate the system in a real environment.

- Optimizing the system, so that it can be incorporated into a "home" environment, which allows us to make it accessible to the general public. 


\section{Bibliographical References}

[BENS00] Shreiderman, Ben. : Universal Usability: Communications of the ACM: Embedding the Internet. May 2000 - Vol. 43 no 5. Page 85

[CLEM99] Clement, A. Shade, L. R.: The Access Rainbow: Conceptualizing Universal access to the information Communications infrastructure. In Gurstein, M., Ed. Community Informatics: Enabling Communities with Information and Communications Technologies. Idea Publishing, Hersey; PA, 1999

[REVE02]Reventós, Laia.: El País: Ciberp@is: Info XXI: Administración.es, casi igual que hace un año. 19 December 2002, Page 5 